\title{
The new tradition - Dialogues on production
}

\section{in architecture}

\author{
M. Ferrari \\ Department of Architecture and Arts, Università Iuav di Venezia, Italy \\ E-mail: margheritaf@iuav.it
}

\begin{abstract}
-
Tools of digitalisation and automation are changing the labour market and human's interaction with production material in general. In architectural processes, this change involves the stage of production but also, and more interestingly, the design one. Architecture has a social role, which includes many processes. Each process requires different times, challenges, and tools. Consequently, the impact of innovation is not the same for these processes. Many manufactories require innovative design and advanced researches. These manufactories have different relationships with digitalisation and innovation, and not all factories have advanced technology; the lack of updates is due to financial or cultural reasons.

This paper aims at investigating the production of structural wood components of the architectural process. The analysis of the impact of innovation in the architectural process cannot be limited to robot or other digital tools, but it is necessary to consider the social and cultural context as well (1). The study is divided in two sections: (2) the role of architecture at the margin of numerous processes (between design and production), but also between innovation and tradition. The investigation needs to include many factors in order to best describe how innovation affects the design and the production. (3) The methodology is structured in three dialogues: with research, design, and manufacturing. Each one of these has specific limits and tools. The collected data describes the role of innovation and tradition in the specific sector of structural wood components of the architectural process.
\end{abstract}

Keywords -

Cultural change; Dialogue; Architecture; Manufacturing.

\section{Introduction}

Technical evolution has always been considered within its social conditions: the cultural and geographical backgrounds influence technological innovation and its impact on people.

Three important truths emerge from this close relationship: the rapid development of digital tools, the gap between different work sectors, and the 'fear' of machines and automation. These conditions are closely related, and each one determines the others.

The rapid development of digital tools improves day by day: the possibility of working from different countries or of sending digital information to line production are only two of many examples.

These digital tools interact with humans in various ways every day, consequently absorbing innovation in different ways as well: the lack of updates can be a discriminating factor, and it can increase the distance between the different work sectors. This is a dangerous gap because it can compromise the entire process, like the complex one of architecture for instance.

The third emerging reality is that of 'fear', deriving from the gap between those who use digitalisation and those who do not. There is a misunderstanding of digital evolution, as it is not considered in accordance with the context in which it develops. Consequently, this fear fuels the rejection of digital change, of evolution, and of the introduction of new work tools. In the course of history, many technical revolutions have evolved the labour market and human's social condition. However, actual technical evolution is different: it is very fast and it influences many aspects of human life (especially work related), its identity, and its cultural tradition.

Therefore, the three emerging truths - rapidity, work gap, fear - concern every work process, and could compromise its actual quality, even in architecture.

This paper aims at investigating the impact of digital innovation on architecture, how new tools are integrated in its processes, especially in the component production 
one. Although the paper focuses the research on this specific process, evaluating the development of other architectural processes is also necessary in order to better understand the development of digital and automation tools. Considerations on a single process cannot be made separately from the others.

The paper is organised in two parts: the first one reconstructs the processes of architecture and it describes the impact of innovation on them. These processes are very different, because each one has its own times and tools. For this reason, communication between these different processes is not simple and the involved factors often encounter difficulties. An architect must be capable of coordinating the numerous factors that move along the margins of the various processes. In this scenario, digital tools could simplify this complex communication and could help to solve some difficulties between the many architectural factors. This section focuses on the interaction between design and production because there is a gap between them that is rapidly expanding. One of the reasons for this is the close relationship between manufactories and their local tradition. In order to investigate the relationship between innovation and tradition, we chose one specific productive process: the structural wood components of the architectural process, because it represents one of the best contexts in which tradition meets innovation. In the architectural context, there are numerous digital tools for design processes on the one side, and many manufactories connected to their traditional tools for wood modelling on the other. The evolution in the design and the production processes necessarily have different speeds: each process involves different kinds of factors and materials. This difference can generate the abovementioned work gap and human fear: in architecture, these correspond to solutions that are less and less adaptable to the social needs. Moreover, productive actions today are not only human or mechanic, but also automated and robotic. The actual processes of modelling and assembly can be expanded beyond human capabilities and its traditional tools. These new limits redefine architectural processes and their relationship with tradition, local materials, and cultural context. The aim of the research is to analyse this specific gap.

The second section of the paper describes the methodology used to investigate main architectural processes: design, research, production, and their levels of innovation. This methodology was structured for the $\mathrm{PhD}$ research in progress at Università Iuav di Venezia. The research deals with the impact of automation on the design of structural wood components, starting from industrial applications, experiments, and failed attempts, up to discovering the new limits that are created by automation and new intelligences.
This requires different investigation strategies, although the challenge is the same: defining the level of innovation and its impact on the building sector.

The analysis allows reconstructing the role of automation in each architectural process and evaluating their interaction. This last aspect is in fact fundamental in order to develop architectural quality, in accordance with the social and functional needs described by Virginia Gangemi [1]: in this case, automation can contribute to the development of an appropriate technological and architectural design.

To understand the appropriation of technology, it is necessary to investigate on more levels. A specific methodology that uses several research tools, in accordance with the different processes and actors, has been developed precisely for this reason. Final data collection helps to compare the role of innovation in each single process: for example, comparing the advanced university research with the manufacturing production, and the innovative automation with the traditional tools. Thanks to these comparisons, it is possible to evaluate how the architectural production process is changing, and consequently how the architectural design process is as well. This research is structured in three dialogues that deal with some of the most important factors of the architectural process: the researches, the designers, and the manufactories. The final comparison can help describe the appropriateness of automation in architecture and in its cultural context.

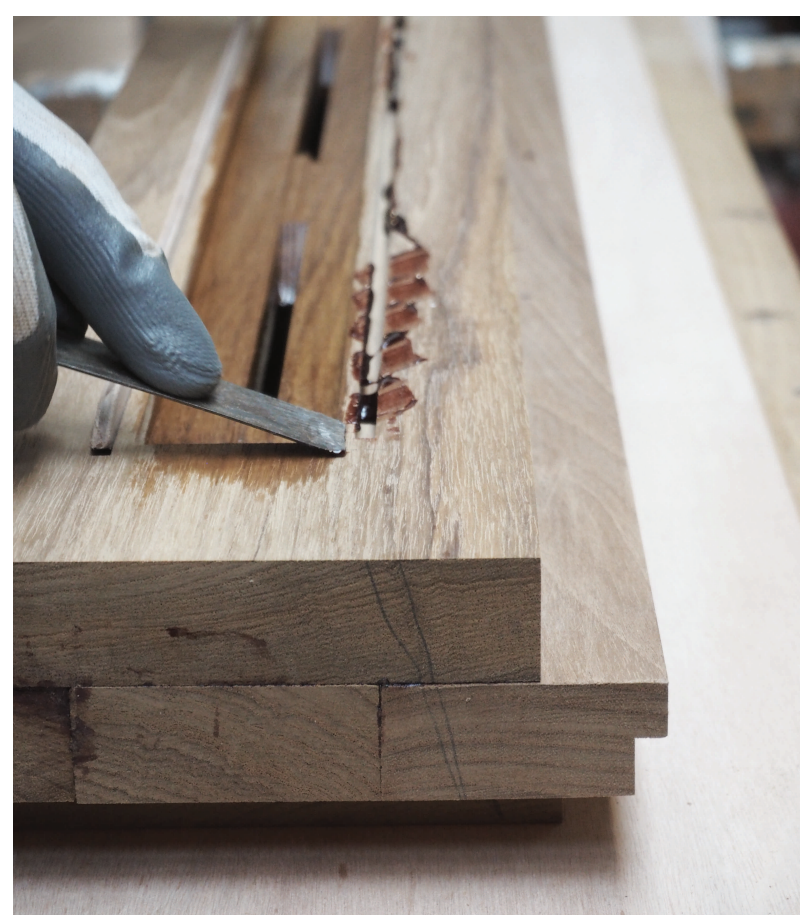

Figure 1. The human action in the processing of a wooden component. 


\section{On the margin}

Invisible technology, as Nicola Sinopoli describes it [2], deals with the complexity of the architectural process and the numerous operators who are involved in it. Digital innovation has a specific type of impact on each process, with important consequences on the communication between the factors involved. In fact, digitalisation and automation allow many operators to communicate in shared digital dimensions through shared languages of informatics. On the other side, the tools of digitalisation and information can amplify the gap between the various architectural processes, like between design and production. Therefore, the interaction between these invisible technologies (in accordance with the integration of digitalisation) can either be very efficient and productive, or bring to architectural and social failure.

The architect is the administrator of these invisible processes, although the complexity of the architectural discipline has grown century-by-century in history: today, there are numerous specialisations in the field, and the architect is required to hold and balance most of them. It is like a sort of 'borderline', a common ground where several professions meet, each with its own challenges, times, tools, and approaches to automation and digitalisation. Today's digitalisation and automation are changing every profession and every profession's processes. The capability of these processes to update themselves weighs in on the balance of the architectural production as a whole. Therefore, the misunderstanding of the digital evolution and the lack of updates could compromise the architectural quality and its construction. The proposed analysis aims to understand the main reasons behind this deficiency: these reasons could be financial or even cultural, where tradition holds an important role.

By investigating along the margins of innovative and traditional production, the paper aims to understand the impact of digital tools on architectural quality, in order to explore and redefine the relationship between men and their architectural means [3], both human and robotic. As according to Stephen Kieran and James Timberlake [4], an architect cannot embody all qualities of a master builder, but thanks to digital tools he can integrate the numerous processes that are involved in the construction project and can coordinate the architectural design in every process and detail. This refers to several architectural aspects and on several scales: the design and the manufacture processes are closely linked and cannot be considered separately from one another. The architect, like an artisan, has the capability of transforming ideas into manufactured matter, modelling it through its intrinsic proprieties. Material production and its tools has a long history, one that has revolutionised the architectural process in time.
The same is happening today: digitalisation and automation influence architectural design and creativity.

The interaction between idea and material matter through digitalisation can contribute to "rebuild" the figure of the manufacturer. As Guido Nardi says [5]: "The choice of materials and the use of particular techniques $[\ldots]$ are strongly conditioned by social, family, and religious traditions, which are the cultural element that is usually internalised, operating in a subconscious dimension. In material civilisations, the manufacturer was the holder of this complex but uniform knowledge. They converge both technical abilities and scientific and social knowing in their manufactured product". Therefore, the architect is a figure that stands on the margin of design and production, but also of tradition and innovation. Today's "contemporary master builder" could resume this care for detail and its production.

The research methodology elaborated for this investigation aims to describe the interaction between architectural processes, as well as how this same interaction can improve the architectural design. The methodology is based on an important definition of innovation and its role. The level of innovation cannot be measured according to one specific technique. Innovation deals with technology, which is the application of a technique in a specific context that holds specific social and cultural needs. Innovation represents a change in a cultural process. Therefore, innovative technology is considered as a cultural process and not as a mere tool. Only a multidisciplinary investigation can make it possible to evaluate how new technologies will be innovative and appropriate for the people, their culture, and their tradition.

\section{Investigation methodology and factors}

Methodologies deal with an array of operators and factors, which interact with automation in many different ways. Therefore, the proposed methodology is based on several tools in order to investigate in different contexts, some more innovative and others more traditional. As said earlier, the sector of the production of structural wood components represents one of the best cases of interaction between traditional and innovative tools.

The architectural process in wood component design and production involves numerous factors and operators, distinguishing three categories:

- $\quad$ researches (from university and companies);

- $\quad$ designers (architects and engineers);

- manufactories (carpentries and joineries).

These factors are respectively associated to three architectural processes: advanced research, architectural 
design, and production. The investigation aims at describing the role of automation and digitalisation in each process, outlining three investigation phases:

- $\quad$ the limits of the survey;

- the construction of investigative tools;

- the data collection.

The three investigation categories (Researches, Designers, Manufactories) are described below and are considered as 'Dialogues': dialogue represents one of the first and most important tools of knowledge, like the ancient example of Platonic Dialogues (IV-III cen. BC); this research also underlines the importance of dialogue in creating an interaction with several realities, like academic research and manufactories. However, there are some useful considerations that must be made in order to devise proper investigation tools: the expected results will be both theoretical and practical, so their comparison could seem difficult, but it presents many common points. The second consideration is the geographic location of the object of the investigation: the cultural context and the financial status contribute (or not) to the development of automation technology.

\subsection{Dialogue 1 - Researches}

Advanced research in automation in architectural processes covers an extensive field that is, however, increasing accessible. Many different figures work on architectural automation, therefore a more selective investigation is necessary.

The specific limits of the survey were structured on the following conditions:

- $\quad$ research unit (both universities and companies);

- $\quad$ research unit with at least 5 years of activity;

- $\quad$ research on structural components, both modelling and assembly;

- $\quad$ research unit in Europe.

In this investigation, wood is not a restricting parameter: a general investigation on automation and structure was preferred to a specific investigation on wood structures. It allows to better understand the interaction between different research fields and their developments. 30 research units were identified in Europe, but only 9 units follow the said parameters. These are generally specialised in specific sectors of architecture or tools: for example, robot or additive manufacturing, solid material modelling or component movement.

The research units were analysed on the basis of progressed researches, which contribute to the definition of a profile for each research unit. The bibliography and the interview were the main tools of investigation in this category.

\subsection{Dialogue 2 - Designers}

The second dialogue is with designers, aiming to form a database of best practices. The selection of architects and engineers began with the identification of case studies. In this category, the limits of the research field refer to buildings (not designers). Each case study had to follow specific parameters:

- it must present an existing building, with environmental and security parameters;

- $\quad$ the building must present a wooden structure;

- $\quad$ it must be localised in Europe;

- $\quad$ its construction must have been completed in the last 10 years;

- the wood component production must have had a significant role in the design process.

The case studies represent the best examples of interaction between design and production. The dialogue between operators is the most important tool that allows the development of the architecture. Digital tools contribute to simplify communication, reducing the margin of error. This communication concerns human and machines, redefining the invisible technology of the architectural processes.

Each case study had to follow specific parameters, with the aim of reconstructing the timeline of the design, production, and assembly processes. Another important parameter was the kind of machine that was used for the wood component production. Thanks to this data, it was possible to identify the most popular machines for architectural production.

\subsection{Dialogue 3 - Manufactories}

The third dialogue deals with manufactories, presenting several complexities according to their variability and their availability to dialogue with research. Analysing this category is important in order to describe the impact of innovation on local economy. In fact, new digital tools can help develop new architectural design processes, on one side, but also redefine the work processes and the role of human workers on the other. Initially, this dialogue aims to evaluate the innovation in the wood production process through the quantity of the machines and robots that are used. The final goal was to create a "map of innovation"; however, many manufactories would necessarily be excluded from this map because of their lack of machines and automation. 


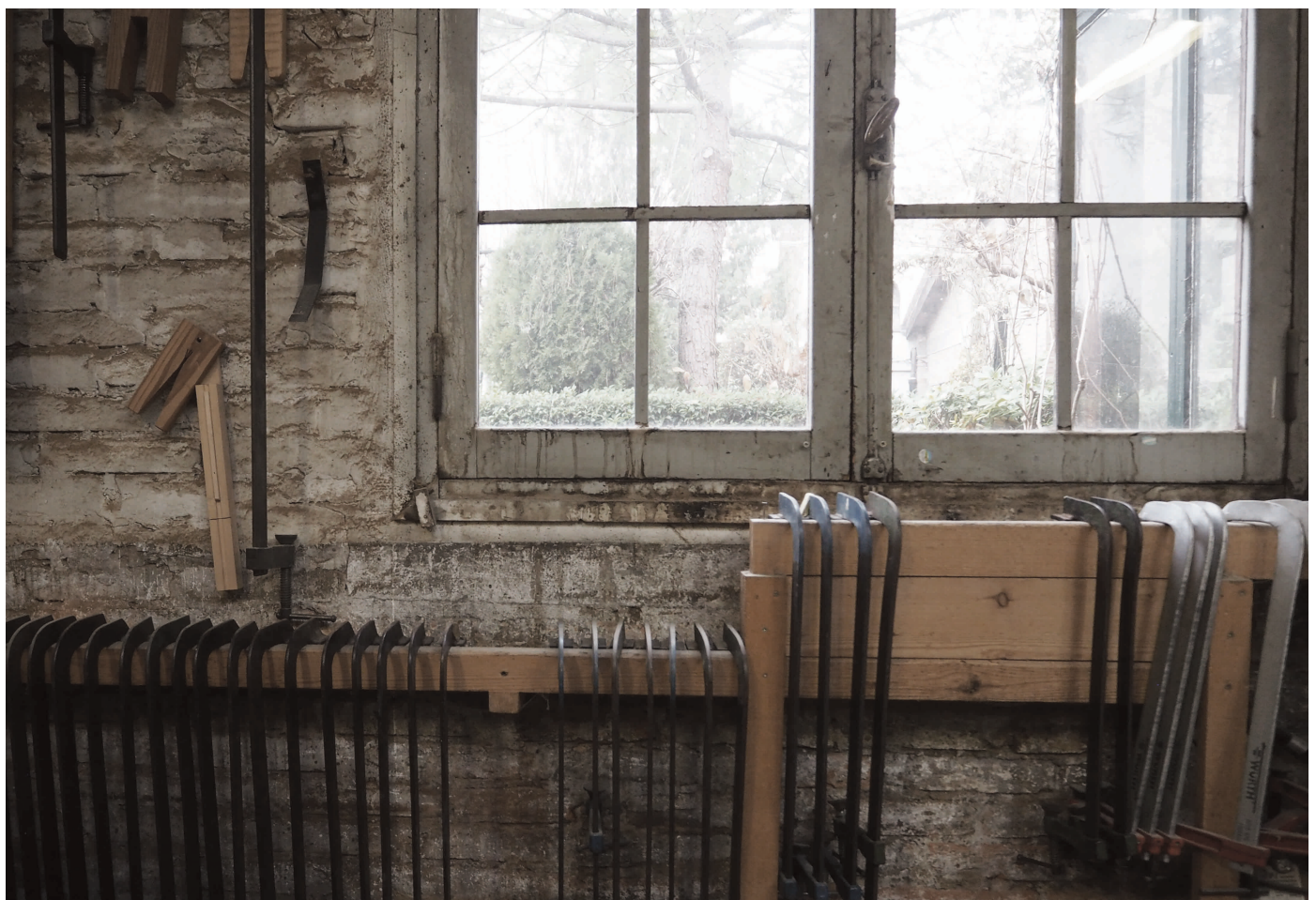

Figure 2. Traditional tools in a Venetian joinery.

As mentioned previously, technological innovation is a process and is not only the result of digital or automated tools: innovation is also conditioned by the social and financial context. Therefore, the geographical location of these manufactories is determinant for their innovation. The investigation became the main tool in discovering the state of the art of manufactories, their variability, and their innovative levels. The analysis was developed in the geographical area of North-East Italy, known as Triveneto, which is composed by three regions: Veneto, Friuli Venezia Giulia, and Trentino Alto Adige. The Triveneto represents one of the most productive areas of Europe and it is characterised by the presences of small and medium businesses with a historical tradition. The parameters for the category were:

- $\quad$ The geographical area (North-East Italy);

- The specific business product, according to the Italian Business Register. Each product was required to have a specific code: $A T E C O$; this research used two ATECO: 16.10 Cutting and planing of wood, 16.23.2 Manufacture of other wood carpentry products and carpentry for construction;

- The manufactory had to be an active business.

Through these parameters, 3.112 businesses were selected, of which 770 carpentries and 2342 joineries. This business database was then ordered according to the specific geography, number of employees, and start year of the business.

A questionnaire was elaborated in order to collect data from the selected businesses, following several levels of investigation. According to the number of participants, thanks to this questionnaire it will be possible to describe the role of automation in wood manufacturing and the role of automation in production.

The questions deal with the presence or absence of digital and automation tools, their use or disuse, and consequently the main reasons behind several production choices. Following this first step, a second step addresses the type of machines and their use for architectural components or material.

In the dialogue with manufactories, the impact of innovation is obviously considered, but the other important common point that is considered is the manufactories' relationship with tradition, their tools, and their materials.

\section{Conclusion}

The research on automated machines and robots in architectural processes needs to consider many various aspects: the languages of communication, the kind of activity, the level of innovation, the update and upgrade status, and the relation with social context. The paper aims at investigating the impact of digitalisation on architecture, starting with the definition of the 
methodology of research.

A multidisciplinary approach was necessary to investigate the complex issue, not only for the nature of the processes and their main tools, but also for their integration with innovation and automation.

Starting from the definition of three emerging truths - rapidity, work gap, fear - the investigation on the role of automation in architecture is necessary to describe how innovation can actually contribute to architectural and social quality as a whole. There is a direct connection between architectural design and production: the research aims to identify a strategic point of view on the issue, in order to develop a knowledge and understanding of technological improvement in architecture. It is possible to set up an investigation at the margins of architectural processes, analysing each one and comparing them.

The research focuses on the production of structural wood components, because this sector represents one of the best interactions between tradition and innovation, since it involves advanced design and research in local manufactories. The manufactories that are considered are joineries and carpentries presenting different levels of innovation. The comparison between the architectural factors and the description of their innovative role is the main tool for a better understating of the appropriation of automation and digitalisation in architecture.

The research identifies three process categories: the research, the design, and the manufacturing. Each one of these processes includes factors with which it dialogues. Therefore, the research methodology is structured and adapted in order to investigate different processes with different research limits.

The research methodology is based on three forms of dialogue: Researches, Designers, and Manufactories. The comparison between these processes concerns the impact of innovation in social culture. Innovation is more than a simple technique: it is a process and a cultural change. The interaction between these three sectors represents a cross-disciplinary description, which is useful to understand if an innovative technology can be appropriate. Although this paper investigates a specific context (structural wood components), the research methodology can be applied to several sectors, which can differ in their architectural and geographical qualities. The aim of the research is the dialogue between many aspects of the architectural process: design and production, human and machine, tradition and innovation. Therefore, dialogue becomes the main investigation tool, able to involve and compare many factors. It can contribute to contrast the human fear toward digital innovation and its rapid evolution. It can help define the role of innovation not only in architecture, but also in society and in the labour market. 'Dialogue' holds the capability of comparing the innovation levels between many processes and their contexts. It is where research and designers come into contact and where (and how) man redefines a new tradition. Comparing research and manufactories, designers and production is necessary to understand the role of automation and digitalisation in architecture. Architecture must be capable of answering social needs and offer a better quality of life. Therefore, dialogue is not an answer but a tool for the analysis of the appropriation of innovation in our society. Innovation is not automation but how we use it. Luca De Biase wrote [6]: "Distinguishing between what is important and what is only interesting, and making the umpteenth cultural leap. Everyone, together. Because even if computers go faster, humans can go farther". Human capability is fundamental in developing new tools and in recognising the cultural change to which they contribute. The research on automated machines and robots in architectural process cannot be made separately from the cultural context, the human workforce and its traditions. Man must continue updating his capabilities and his territorial knowledge: thanks to these, he can use innovative tools like automation and digitalisation to increase his potential and his culture. He can redefine tradition: he can build a new tradition, a new relationship with materials through new tools. If not, if technique remains the main point of innovation, man will inevitably become and remain only a spectator.

\section{References}

[1] Gangemi V. (Edited by). Architettura e tecnologia appropriata. Franco Angeli, Milano, 1985.

[2] Sinopoli N. La tecnologia invisibile. Franco Angeli, Milano, 1997.

[3] Picon A. Robots and architecture. Experiments fiction epistemology. AD Architectural Design, 84(3): 54-59, 2014.

[4] Kieran S. and Timberlake J. Refabricating architecture. McGraw-Hill, New York, 2004.

[5] Nardi G. Le nuove radici antiche. Franco Angeli, Milano, 1994, page 37.

[6] De Biase L. Homo pluralis. Essere umani nell'era tecnologica. Codice Edizioni, Torino, 2016. 